\title{
Mucormycosis: Botanical Insights Into The Major Causative Agents
}

\section{Naser A. Anjum}

Department of Botany, Aligarh Muslim University, Aligarh-202002 (India).

e-mail:dnaanjum@gmail.com; naanjum1@myamu.ac.in; anjum@ua.pt

SCOPUS Author ID: 23097123400

https://www.scopus.com/authid/detail.uri?authorId=23097123400 


\begin{abstract}
Mucormycosis (previously called zygomycosis or phycomycosis), an aggressive, life-threatening infection is further aggravating the human health-impact of the devastating COVID-19 pandemic. Additionally, a great deal of mostly misleading discussion is focused also on the aggravation of the COVID-19 accrued impacts due to the white and yellow fungal diseases. In addition to the knowledge of important risk factors, modes of spread, pathogenesis and host defences, a critical discussion on the botanical insights into the main causative agents of mucormycosis in the current context is very imperative. Given above, in this paper: (i) general background of the mucormycosis and COVID-19 is briefly presented; (ii) overview of fungi is presented, the major beneficial and harmful fungi are highlighted; and also the major ways of fungal infections such as mycosis, mycotoxicosis, and mycetismus are enlightened; (iii) the major causative agents of mucormycosis are critically discussed; (iv) the systematic position, occurrence, structure, and the major modes of reproduction are summarized for Rhizopus sp. and Mucor sp., the major causative agents of mucormycosis; (v) the other causative agents of mucormycosis includes Cunninghamella bertholletiae, Apophysomyces elegans, Lichtheimia (Absidia), Saksenaea, and Rhizomucor pusillus are briefly introduced; (vi) facts related with the infection-misnomers based on fungi colour are also highlighted; and lastly, (vii) the major take-home message and clues for future research on the subject are listed.
\end{abstract}

Keywords Mucormycosis; Causative agents; Rhizopus sp.; Mucor sp.; Botanical insights; COVID-19 


\section{CONTENTS}

\section{Introduction}

2. Fungi - an overview

2.1 Beneficial fungi

2.2 Harmful fungi

2.2.1 Mycosis

2.2.2 Mycotoxicosis

2.2.3 Mycetismus

\section{Mucormycosis}

3.1 Rhizopus

3.1.1 Systematic position

3.1.2 Occurrence

3.1.3 Structure

3.1.4 Reproduction

3.1.4.1 Vegetative reproduction

3.1.4.2 Asexual reproduction

3.1.4.3 Sexual reproduction

3.2 Mucor

3.2.1 Systematic position

3.2.2 Occurrence

3.2.3 Structure

3.2.4 Reproduction

3.2.4.1 Vegetative reproduction

3.2.4.2 Asexual reproduction

3.2.4.3 Sexual reproduction

3.3 Cunninghamella bertholletiae

3.4 Apophysomyces elegans

3.6 Lichtheimia (Absidia)

3.7 Saksenaea

3.8 Rhizomucor pusillus

\section{Infection-misnomers based on fungi colour}

\section{Conclusion and perspective}

\section{References}




\section{Introduction}

The world is already struggling against a rapid increase in the coronavirus disease (COVID-19), an infectious disease caused by a newly discovered coronavirus called SARS-CoV-2. Since China reported its first cases to the World Health Organization (WHO) in December 2019, as of May 25, 2021, authorities in 219 countries and territories have reported about 167.4 million COVID-19 cases and 3.5 million deaths (CNN Health 2021). The impact of COVID-19 in human is further aggravated by the incidence of another life-threatening fungus-caused disease known as the mucormycosis (previously known as zygomycosis or phycomycosis; 'black fungus' infection).

Mucormycosis, a devastating invasive fungal infection with high mortality rates is caused by ubiquitous filamentous saprophytic fungi (Order: Mucorales; Class: Mucormycetes). The order Mucorales is the largest and the best-studied order of zygomycete fungi. Notably, broad, aseptate or sparsely septate ribbon-like hyphae are exhibited by the members of order Mucorales. Members of the order Mucorales are ubiquitous, and most species are chiefly terrestrial saprophytes. Decaying plant or animal matter rich in starch and sugars are mostly preferred as the major growing substrate. Mucorales are familiarly known as the 'black molds'; and a few species from this order cause human and animal disease zygomycosis, presently called as mucormycosis (Agrawal et al. 2020).

Rhizopus species are the major culprit of mucormycosis, which is followed by Mucor species and Cunninghamella bertholletiae, Apophysomyces elegans, Lichtheimia (Absidia) species, Saksenaea species, and Rhizomucor pusillus. Mucormycosis can involve many different organs, including the skin, nasal sinuses, orbit, and brain (rhino-orbital-cerebral), lung, and gastrointestinal tract. Moreover, the list of common risk factors for mucormycosis includes diabetes mellitus, hematological malignancies, solid-organ transplantation, and other immunosuppressive conditions (Dannaoui and Millon 2014). Compared to 
Europe and United States, the prevalence of mucormycosis appears to be disproportionately high in patients with uncontrolled diabetes in India (Prakash et al. 2016). However, mucormycosis is being reported in a few COVID-19 patients (during or post-recovery) in most Indian states including Delhi, Maharashtra, Gujarat and Rajasthan. As of $26^{\text {th }}$ May 2021, 11,717 cases of black fungus have been reported, where Gujarat $(2,859)$ has most cases (NDTV 2021).

In addition to the 'black fungus' infection (mucormycosis), a great deal of discussion is currently focused in the world media/news on the aggravation of the COVID-19 accrued impacts due to the white and yellow fungal diseases. However, most of the information to this end is misleading that can create havoc in the public, and may also make the impact of COVID-19 and the major fungal infections in human grimmer. Additionally, the major science underlying fungibased infections including black, white and yellow fungal diseases is clearly meager.

Given above, discussion in this paper aims to: present an overview of fungi; highlight the major beneficial and harmful fungi; enlighten the major ways of fungal infections such as mycosis, mycotoxicosis, and mycetismus; critically discuss the major causative agents of mucormycosis; summarize the systematic position, occurrence, structure, and the major modes of reproduction for Rhizopus sp. and Mucor sp., the major causative agents of mucormycosis; briefly introduce the other causative agents of mucormycosis includes Cunninghamella bertholletiae, Apophysomyces elegans, Lichtheimia (Absidia), Saksenaea, and Rhizomucor pusillus; and also to highlight the major facts related with the infection-misnomers based on fungi colour. Finally, the major take-home message and clues for future research on the subject are summarized. 


\section{Fungi - an overview}

The group 'Fungi' (singular: fungus) can be defined as heterogenous assemblage of 'achlorophyllous thallophytes'. In other words, fungi are living (saprophytic and parasitic spore-producing) organisms and lack chlorophyll. Additionally, fungi have chitin-containing cell walls, a polysaccharide found also in insect exoskeletons. Fungi may be unicellular, syncytial (many nuclei not separated into different cells) and multicellular (nuclei separated by septa). The group fungi possess chromosomes within membrane-bound nuclei, dividing through mitosis. The Kingdom Fungi is purely dedicated to fungi. About 100,000 species of fungi have been identified to date. Additionally, high-throughput sequencing methods suggested the existence of as many as 5.1 million fungal species on Earth. Fungal diversity harbour almost all regions (habitats) on Earth, and have their unique ability to withstand extremes in temperature, water activity, and carbon source. The highest diversity for most fungi occurs in the Tropical regions of the world (Blackwell 2011).

Yeasts and molds are the two main forms of fungi. Yeasts (such as Candida spp. and Cryptococcus spp.) are solitary cells that reproduce by budding. Molds, such as Aspergillus, form multicellular hyphae and can grow by apical extension. Contingent to the temperature and environment, there occur dimorphic (intermediary) fungi that exhibit the phenotype of both (yeast and mold) morphologies. The list of dimorphic fungi includes Histoplasma spp., Coccidioides spp., Blastomyces dermatitidis, Paracoccidioides spp., and Sporothrix spp (Tan and Seng 2018).

\subsection{Beneficial fungi}

Fungi are mostly friend to most ecosystems and the humans. Fungi contribute in balancing most ecosystems by acting as the major decomposers and thereby driving the biogeochemical cycles. This is possible because soils act as an important habitat of high fungal diversity being highest near organic material such as roots and root exudates. The association of fungi with plants is widely 
known. The symbiotic fungi (or mycorrhizae; myco $=$ fungus, $r$ hizo $=$ root) are known to inhabit the roots (of most vascular plants) and supply essential nutrients. Up to $80 \%$ of all plant species and $92 \%$ of plant families are known to harbor arbuscular mycorrhizae (AM) and ectomycorrhizae (outside mycorrhiza), two main types of mycorrhizal fungi. Lichen-forming fungi are contributed by about $20 \%$ of all fungi and $40 \%$ of the ascomycetes $(13,500$ species) (Lutzoni and Miadlikowska 2009). In addition to being used in wine and beer industries, and bioremediation technology, the genus Penicillium is one of fungi that have various plant-growth-promoting characteristics. Penicillium mold naturally produces the antibiotic penicillin. The red bread mold Neurospora crassa and the yeast, Saccharomyces cerevisiae (baker's yeast) are important model research organisms. Some types of fungi (mushrooms: morels, shiitake mushrooms, chanterelles, and truffles, and the humble meadow mushroom, Agaricus campestris) are edible and thereby provide nutrients. Fungi can also be used as microbial insecticides. The fungus Beauveria bassiana is being tested as a possible biological control/ a pesticide for the recent spread of emerald ash borer.

\subsection{Harmful fungi}

Fungi may be parasites, pathogens, and, in a very few cases, predators. Fungal plant pathogens cause devastating disease in crops, and thus are responsible for generating significant losses in yield, quality and quantity in agronomical, horticultural, floricultural and ornamental, and forest plant species worldwide. The list of most common diseases caused by plant pathogenic fungi includes anthracnose, blight, canker, damping off, dieback, gall, leaf spot, powdery mildew, rust, root rot, scab, and wilt (Hariharan and Prasannath 2021).

Fungi can also act as parasites and pathogens in animals including humans. In context with fungal infections in humans, out of so far identified over 1.5 million species of fungi only a small fraction (approximately 300) is known to cause 
disease in humans. Notably, three major ways through which fungi can affect animals, including humans comprise mycosis, mycotoxicosis and mycetismus. These three major ways are briefly elaborated hereunder; however, a great deal of detailed below sections and sub-sections are dedicated to mucormycosis.

\subsubsection{Mycosis}

In mycosis, a pathogenic fungal disease happens due to infection and direct damage. Herein, a successful attack of fungi on the hosts occurs and eventual direct colonization and destruction of their tissues happens. Mycosis-causing pathogenic fungi include mucormycosis, dermatophytoses, yeast infections, systemic mycoses, and mycetoma. Notably, mycoses may be mainly of three types namely superficial, systemic and/or opportunistic. The spread of fungi to the underlying tissue rarely occur in superficial mycoses of the epidermis, hair, and nails. On the other hand, fungi commonly enter the body through the respiratory system in systemic mycoses; and the spread of fungi to internal organs occurs. Some fungi are common in all environments; exhibit low inherent virulence; and constitute an almost limitless number. These fungi actually are the causal agents of opportunistic mycoses infections. After candidiasis and aspergillosis, the mucormycosis is considered as the third most common opportunistic fungal infection; and is caused by diverse fungal organisms in the order Mucorales (including those in the genera Rhizopus, Rhizomucor, Absidia and Mucor) (Revankar 2021).

\subsubsection{Mycotoxicosis}

Numerous fungi can also produce toxic secondary metabolites (such as mycotoxins) often only under specific conditions of warmth, moisture and humidity. Field grains, damaged grains or improperly stored feeds may usually exhibit the development of certain mycotoxins. Hence, ingestion of mycotoxinsloaded grains or forage can cause mycotoxicosis. Over 200 mycotoxins have been identified to date. Mycotoxins such as aflatoxins, ochratoxin A, patulin and toxins 
produced by Fusarium fungi are among 12 mycotoxins that have gained the most attention due to their severe effects on human health and their occurrences in food (WHO 2018). Produced by certain moulds (Aspergillus flavus and Aspergillus parasiticus), aflatoxins are amongst the most poisonous mycotoxins and cause aflatoxicosis. Several species of Aspergillus and Penicillium produce ochratoxin A, which is a common food-contaminating mycotoxin. On the other hand, a variety of moulds, particularly Aspergillus, Penicillium and Byssochlamys; and species of Stemphylium, Alternaria, Fusarium, Trichoderma, Trichothecium, Mucor, and Phialophora produce patulin, highly toxic to plant and animal cells. In fact, patulin can react with terminal sulfhydryl groups of proteins and polypeptides present in foods; has an immunosuppressive effect and inhibits DNA synthesis (Kharayat and Singh 2018).

\subsubsection{Mycetismus}

Mycetismus is the fungal poisoning caused by the ingestion of certain species of potentially deadly mushrooms. Of the 5000 known species of mushrooms, there are only approximately one hundred known to be poisonous. For example, Amanita phalloides (death cap), A. verna, A. virosa (the destroying angel), and certain other species that contain poisons/neurotoxins such as amatoxins (amanitins) (Smith and Davis 2016; Bio Libretexts 2021).

\section{Mucormycosis}

As mentioned also above that mucormycosis is a devastating invasive fungal infection with high mortality rates. This infection is caused by ubiquitous filamentous saprophytic fungi of the Order - Mucorales and Class Mucormycetes). Order Mucorales is the largest and the best-studied order of zygomycete fungi. Notably, broad, aseptate or sparsely septate ribbon-like hyphae are exhibited by members of the order Mucorales. Members of the order Mucorales are ubiquitous, and most species are chiefly terrestrial saprophytes. Decaying plant or animal matter rich in starch and sugars are mostly preferred as the major growing substrate. Mucorales are familiarly known as the 'black 
molds'; and a few species from this order cause human and animal disease zygomycosis, presently called as mucormycosis (Agrawal et al. 2020).

The order Mucorales includes 11 families, 56 genera, and approximately 300 species. Among these, 11 genera and $\sim 27$ species are associated with human infections. Among $>900$ reported cases of mucormycosis, $47 \%$ of the cultureconfirmed mucormycosis was caused by Rhizopus species. On the other hand, Mucor species and Cunninghamella bertholletiae caused $18 \%$ and $7 \%$ mucormycoses respectively. Apophysomyces elegans, Lichtheimia (Absidia) species, and Saksenaea species caused 5\% each causes of mucormycoses. Rhizomucor pusillus was the cause of $4 \%$ of the total cases of mucormycoses analyzed. A variety of other uncommon species represented the remaining $8 \%$ of cultured confirmed cases (Gomes et al. 2011; Kontoyiannis and Lewis 2016; Prakash and Chakrabarti 2019) (Fig. 1).

Gradually increasing emergence of mucormycosis is being reported globally and particularly in developing countries including India. Notably, the prevalence of mucormycosis in India is about 80 times the prevalence in developed countries. Globally, Rhizopus, Mucor and Lichtheimia (formerly Absidia or Myocladus) spp. represented the most frequent (about 70-80\%) causative agents of mucormycosis. However in terms of the specificity to the mucormycosiscausative agents regions worldwide, the predominant causal agents of mucromycosis in Asia and Europe are Apophysomyces variabilis and Lichtheimia species, respectively. Asia has also reported to the evidence of the new causative agents namely Rhizopus homothallicus, Mucor irregularis, and Thamnostylum lucknowense. Additionally, among the Rhizopus sp., Rhizopus oryzae (R. arrhizus) is the most common agent isolated worldwide. Particularly in India, $R$. oryzae ( $R$. arrhizus) and Apophysomyces elegans are respectively the first and the second most common causative agents of mucormycosis (Chakrabarti and Singh 2014; Prakash and Chakrabarti 2019; Skiada et al. 2020). 

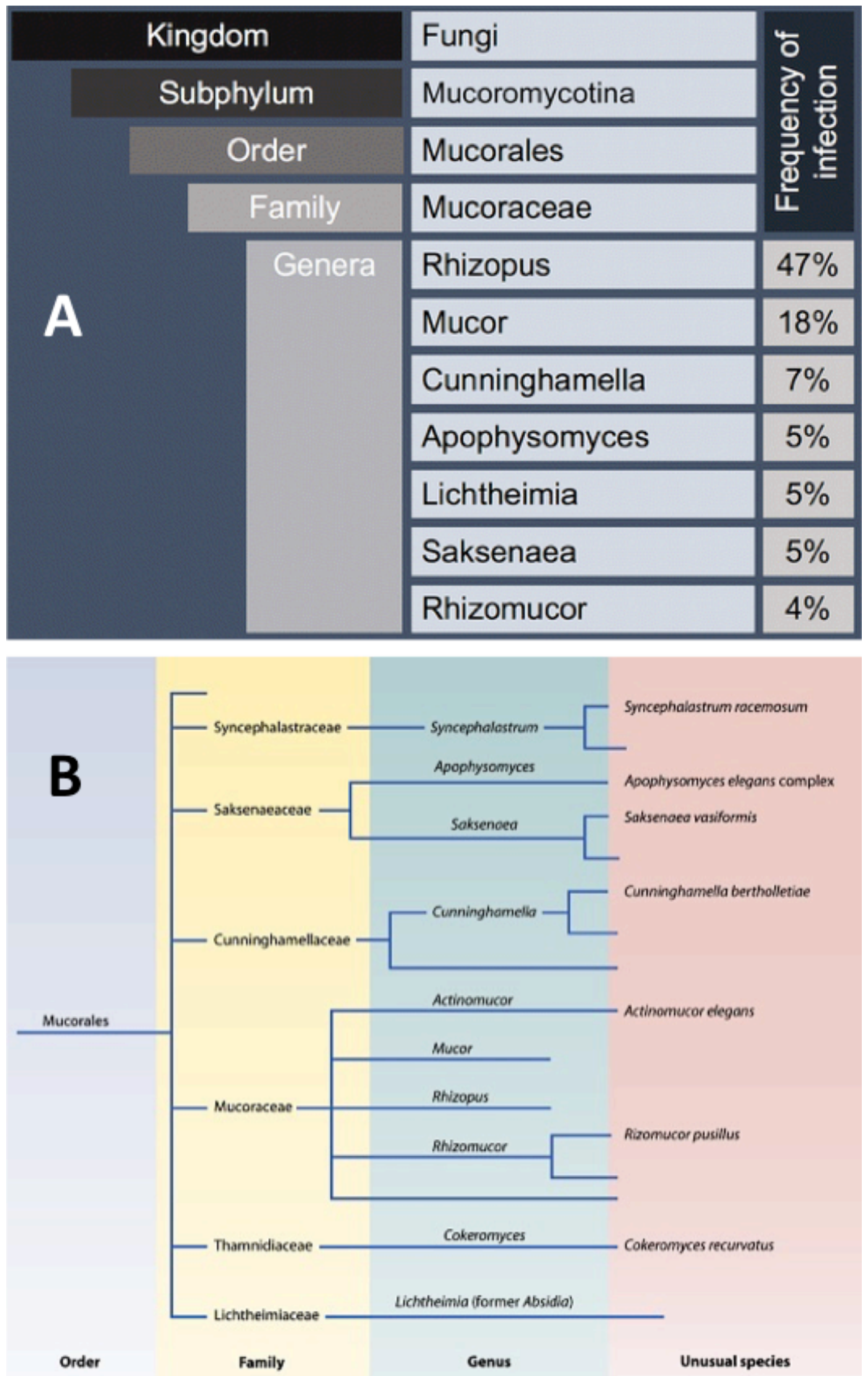

Fig. 1. Taxonomic hierarchy of the genera that most commonly cause mucormycosis (A) (Agrawal et al. 2020), and the taxonomic scheme of humanpathogenic Mucormycetes of the order Mucorales (B) (Gomes et al. 2011). 
Efforts are made herein to provide the readers important botanical details of the major mucormycosis-causative agents including Rhizopus species, Mucor species, Cunninghamella bertholletiae, Apophysomyces elegans, Lichtheimia (Absidia) species, Saksenaea species, and Rhizomucor pusillus.

\subsection{Rhizopus}

The genus Rhizopus has several species (8-10) including Rhizopus caespitosus, $R$. delemari, $R$. homothallicus, $R$. microsporus, $R$. oryzae (now R. arrhizus), R. reflexus, R. schipperae, and R. stolonifer (black bread mold/ the common bread mold).

\subsubsection{Systematic position}

Rhizopus (Black mold of bread) belongs to Division - Mycota (Mycophyta; Fungi), Class - Zygomycetes (Phycomycetes), Order - Mucorales and Family Rhizopodaceae (formerly Mucoraceae).

\subsubsection{Occurrence}

Rhizopus is a filamentous, cosmopolitan and multicellular saprophytic fungus, most commonly growing on dead/decaying organic matter such as vegetables, pickles, neglected leather shoes (during rainy season), fruits, bread, jellies, peanuts, and tobacco etc.

\subsubsection{Structure}

Mycelium (the vegetative structure) is cottony white and narrow, and is made up of multinucleated (coenocytic), aseptate and branched hyphae growing on the surface of the substratum. Actually, mycelium is cottony white only in early stages of growth, and turns brown (or blackish) due to development of zygospores. Both aerial hyphae and prostrate hyphae are clearly differentiated in mycelium. The prostrate hyphae are actually the absorptive (and rhizoidal) hyphae, produce rhizoids at certain intervals, and remain on the surface of and 
penetrate into the substratum. Two groups of hyphae are connected with a structure known as stolon, which grows on the substratum (Fig. 2).

Sporangiophores are the aerial, branched and reproductive mycelia arising from the upper surface of the nodes of the stolon. Terminally, sporangiophores give rise to swollen structure called sporangium filled with spores (sporangiospores).

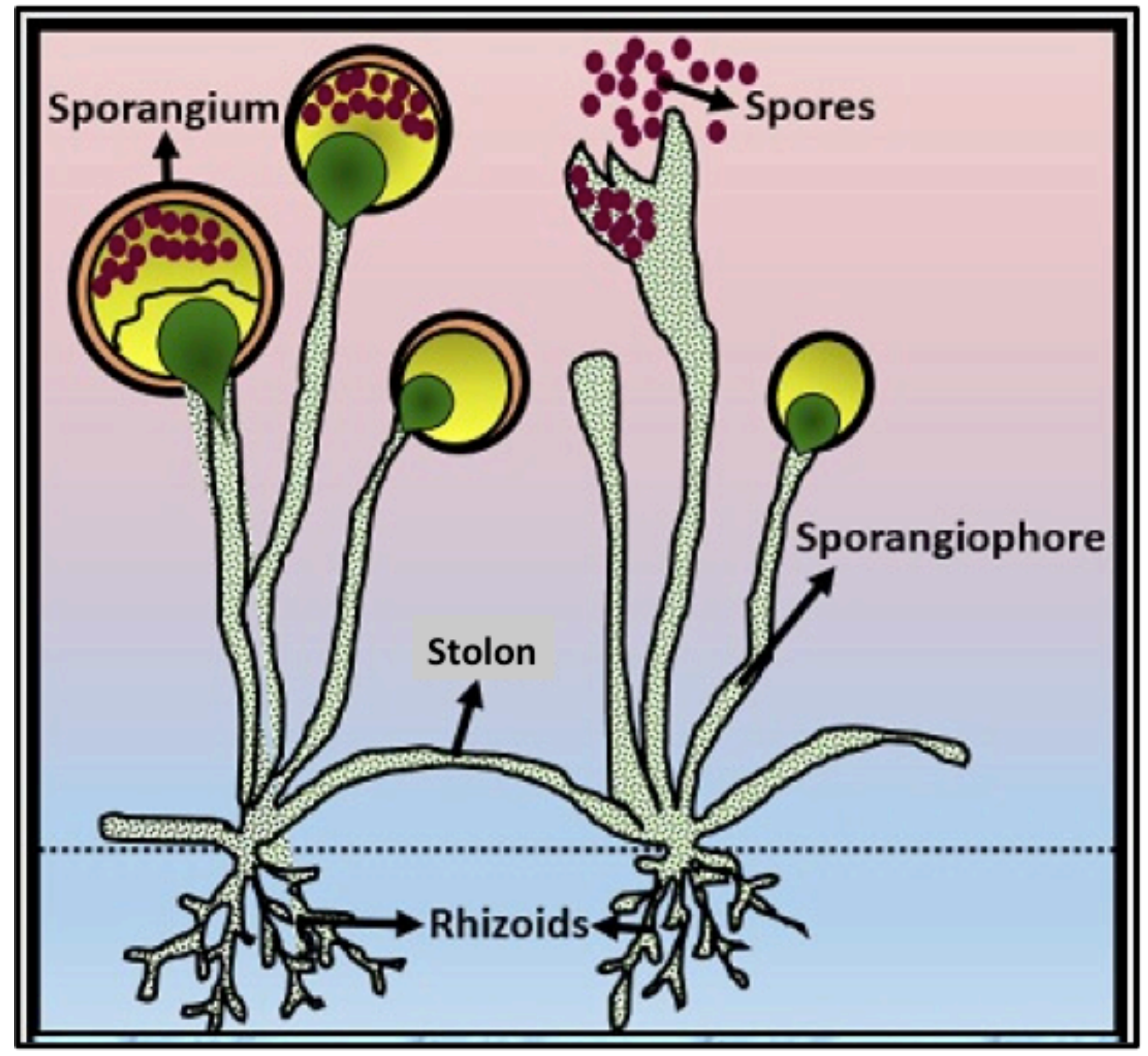

Fig. 2. General structure of Rhizopus species (Supriya 2021a).

\subsubsection{Reproduction}

Vegetative, asexual and sexual modes of reproduction occur in Rhizopus. 


\subsubsection{Vegetative reproduction}

The vegetative mode of reproduction may occur as a result of accidental breaking of hyphae (fragmentation), and/or via formation of chlamydospores, formed under unfavourable conditions such as drought. A complete mycelium is separately developed from each of the fragments of a stolon.

\subsubsection{Asexual reproduction}

Asexual reproduction takes place by the formation of non-motile and multinucleated spores (sporangiospores) formed by cleavage inside a large globose sporangium, a terminal structure of long and erect sporangiophore. During the maturity of the sporangium, a water-absorption-accrued pressure is created in the dome-shaped columella that in turn leads to the bursting of its thin wall and eventual release/dispersal of spores happens. Favourable conditions help spores (sporangiospores) to germinate, produce germ tube and the formation of a new mycelium (Fig. 3).

\subsubsection{Sexual reproduction}

Sexual reproduction occurs by fusion of two compatible hyphae, where multinucleate gametangia (aplanogametangia) are fused (copulated). Except few homothallic (dioecious) species (such as R. sexualis) most of the Rhizopus species are heterothallic and have different mycelium for male (+) and female (-) mating strains. It is important to note here that both gametangia (aplanogametangia) are morphologically (externally) similar but are physiologically different (i.e. + and ), the phenomenon is termed as heterothallism. Mature zygospore (the resting structure) possess 5-layered wall ( 2 in exospore and 3 in endospore). After a rest period, zygospore germinates by meiosis, where exosporium cracks and endosporium produces germsporangiosphore that develops germsporangium terminally. The germsporangium bears only one type of spore (either + or - ), which germinates to form new mycelium (Majumdar and Banerjee 2000; Kaushik 2014) (Fig. 3). 

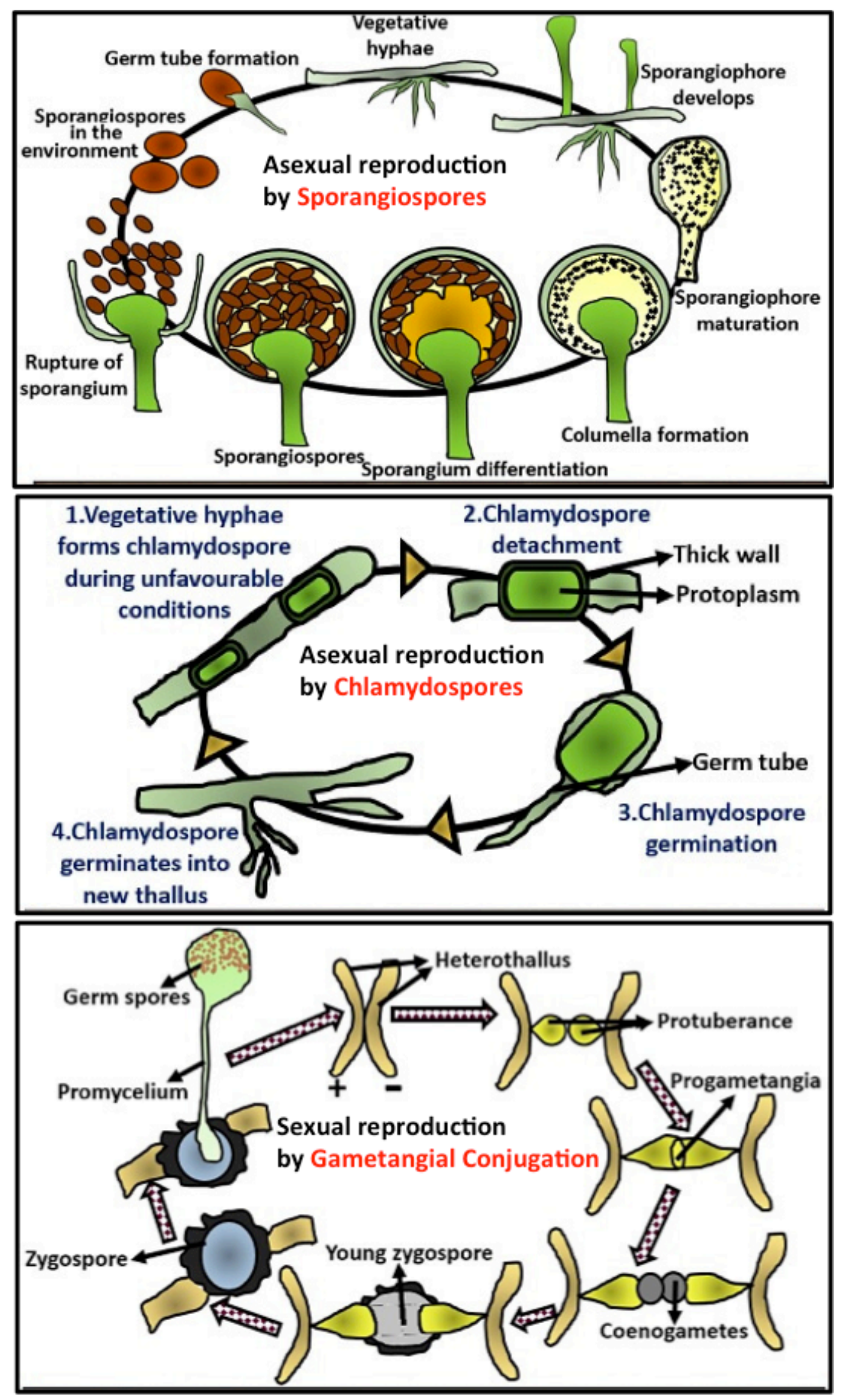

Fig. 3. Major modes of reproduction in Rhizopus species (Supriya 2021a). 


\subsection{Mucor}

Several species of Mucor stand next to Rhizopus sp. in terms of causative agents of mucormycosis. Generally considered as the first saprophytic colonizers on dead or decaying plant material, Mucor as well as many other mucoralean fungi rapidly utilize the limited number of simple sugars (carbohydrate) and thereby dominate the decomposition process (Botha and du Preez 1999; Botha and Botes 2014). Mucor sporangiophores and sporangia resemble a collection of pins. Therefore, Mucor is commonly known as pin mold.

\subsubsection{Systematic position}

Mucor belongs to Division - Mycota (Mycophyta), Class - Zygomycetes, Order Mucorales and Family - Mucoraceae. The genus Mucor comprises $>50$ species including M. amphibiorum, M. circinelloides, M. ellipsoideus, M. fragilis, M. hiemalis, M. hiemalis f. silvaticus, M. indicus, M. mucedo, M. paronychius, M. piriformis, M. plumbeus, M. pseudolusitanicus, $M$. racemosus, $M$. ramosissimus, $M$. variicolumellatus and M. velutinosus..

\subsubsection{Occurrence}

Mucor is a saprophytic fungus and cosmopolitan in distribution. Most species of Mucor grow on decaying organic substances including moist bread, soil and dung, rotten fruit, and also on decaying plant and animal matters. In particular, Mucor species such as M. strictus and M. flavus grow in soil. M. mucedo is a coprophilous species and grows on dungs of herbivorous animals like cow etc. Temperature range of $25-30^{\circ} \mathrm{C}$ is generally suitable for a rapid growth of the Mucor-colonies; however, M. indicus (an aromatic species) may grow at temperatures as high as $40^{\circ} \mathrm{C}$. On the other hand, poor or no growth was noted at $37^{\circ} \mathrm{C}$ for $M$. racemosus and $M$. ramosissimus. 


\subsubsection{Structure}

White, delicate and cotton-like threads, collectively known as mycelium, which spread in all directions over the substratum, represent the body of Mucor. Individual thread is called hyphae which possess chitinous wall and is always much branched and coenocytic i.e. aseptate and multinucleate. However, the bases of the reproductive organ and occasionally the older hyphae possess septa. The hyphae are usually prostrate; however, some of the hyphal branches penetrate down into the substratum; and serve the function of both anchorage, and absorption of water and nutrients (Fig. 4).

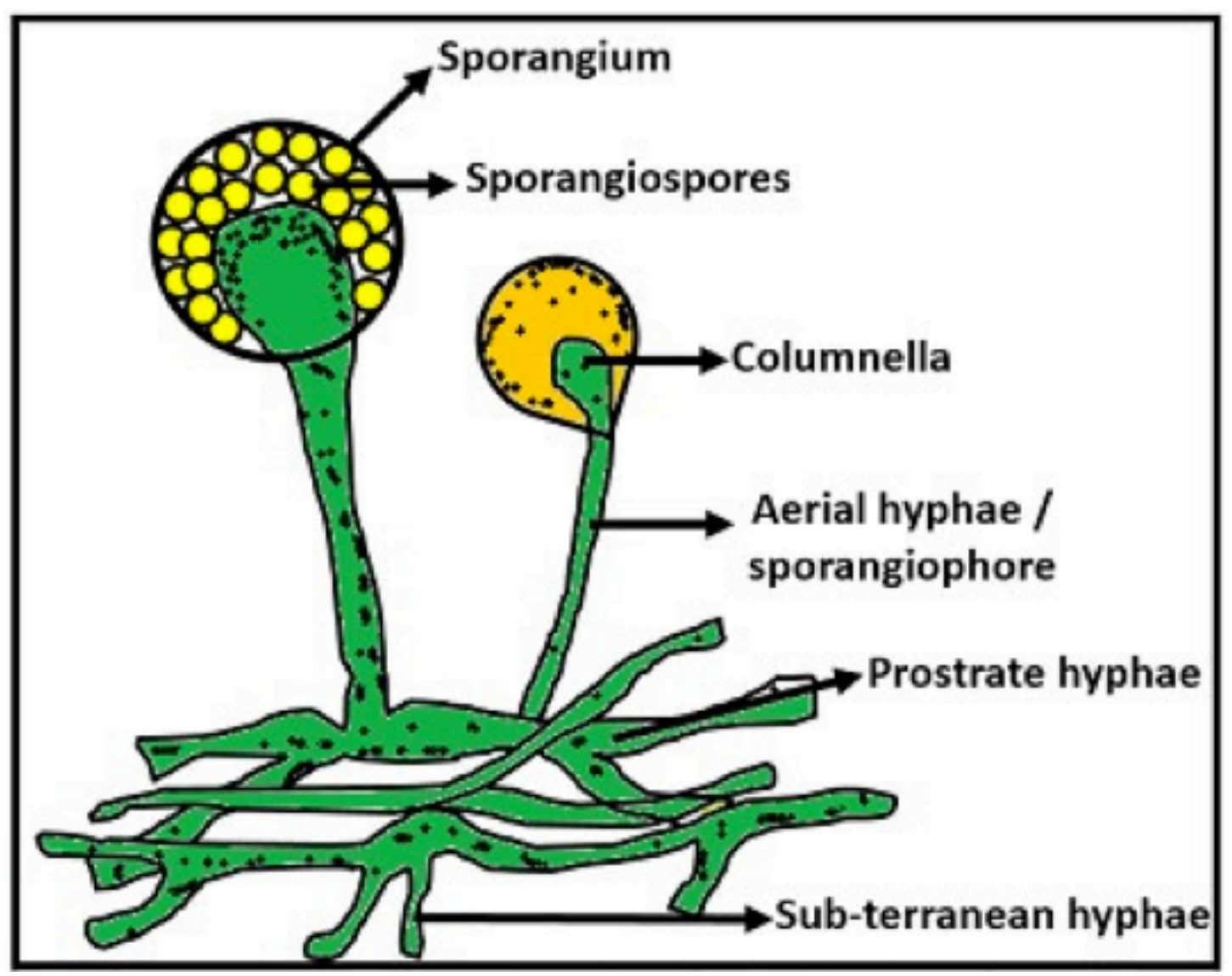

Fig. 4. General structure of Mucor species (Supriya 2021b).

\subsubsection{Reproduction}

Mucor reproduces by vegetative, asexual and sexual modes. 


\subsubsection{Vegetative reproduction}

As happens in most Rhizopus species, the vegetative reproduction in Mucor species takes place by fragmentation as a result of accidental breakage. Two or more units of mycelium (and eventually the mother mycelium) may be formed on breaks up of the mycelium (Fig. 5).

\subsubsection{Asexual reproduction}

Asexual reproduction takes place by the formation of sporangiospore, chlamydospore and sometimes oidia (arthrospores/ oidiospores) (Fig. 5).

\subsection{Sporangiospore formation}

The formation of sporangiospore (or aplanospores; non-motile) occurs during favourable condition. Herein, somatic hyphae (unbranched and slightly broader then other) gives rise to vertical hyphae, which is known as sporangiophore. Sporangiophores are short, erect, and taper towards their apices, and may form short sympodial branches. Notably, cytoplasm gets accumulated in the tip of sporangiophore leading to the formation of sporangia, which are swollen and spherical sac-like structure. Gray to black in color, sporangia is round and 50-300 $\mu \mathrm{m}$ in diameter, and is filled with multinucleate sporangiospores. The wall bursts/ruptures and results into the release of sporangiospores, Mostly insects or wind help in dissemination and free spread of sporangiospores. On getting a suitable substratum, the sporangiospore germinates to form a hypha (Fig. 5).

\subsection{Chlamydospore formation}

Under unfavourable condition, the break-up of transverse wall in some of the hyphae results into the formation of the chains of nutrition-rich and thick-walled cells, which latter round off, and represent chlamydospores. However, in favourable condition, a new mycelium is produced on the germination of chlamydospore (Fig. 5). 
Vegetative reproduction
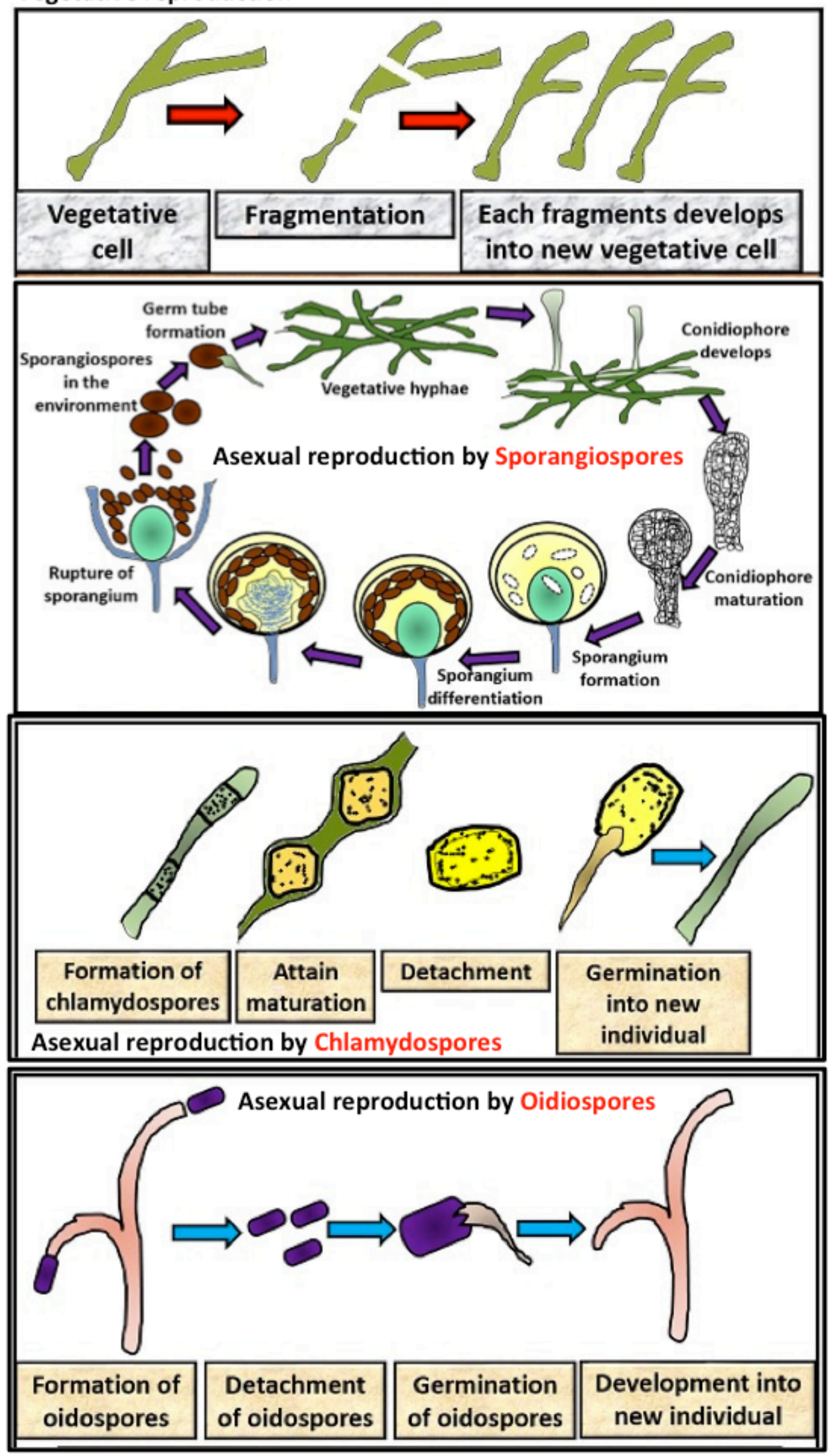

Fig. 5. Vegetative and asexual reproduction in Mucor species (Supriya 2021b). 


\subsection{Oidia formation}

Herein the formation of thin walled bid-like oidia (oidiospore or asexual spore) occurs as a result of a transverse breakage of hyphae; however, in sugar-rich medium under anaerobic condition and in presence of $\mathrm{CO}_{2}$. The detached oidia pass through torula stage and eventually develop into mycelia (Fig. 5).

\subsubsection{Sexual reproduction}

The sexual mode of reproduction in Mucor species occurs at the end of growing season. Herein, the sexual reproduction occurs as a result of gametangial conjugation, which may be brought about by the fusion of two identical gametes (isogamous) involving hypha of either same species (homothallic; e.g., M. tenuis and M. genereosis) or hypha of opposite strain (heterothallic; e.g., M. mucedo and M. hiemalis) (Fig. 6).

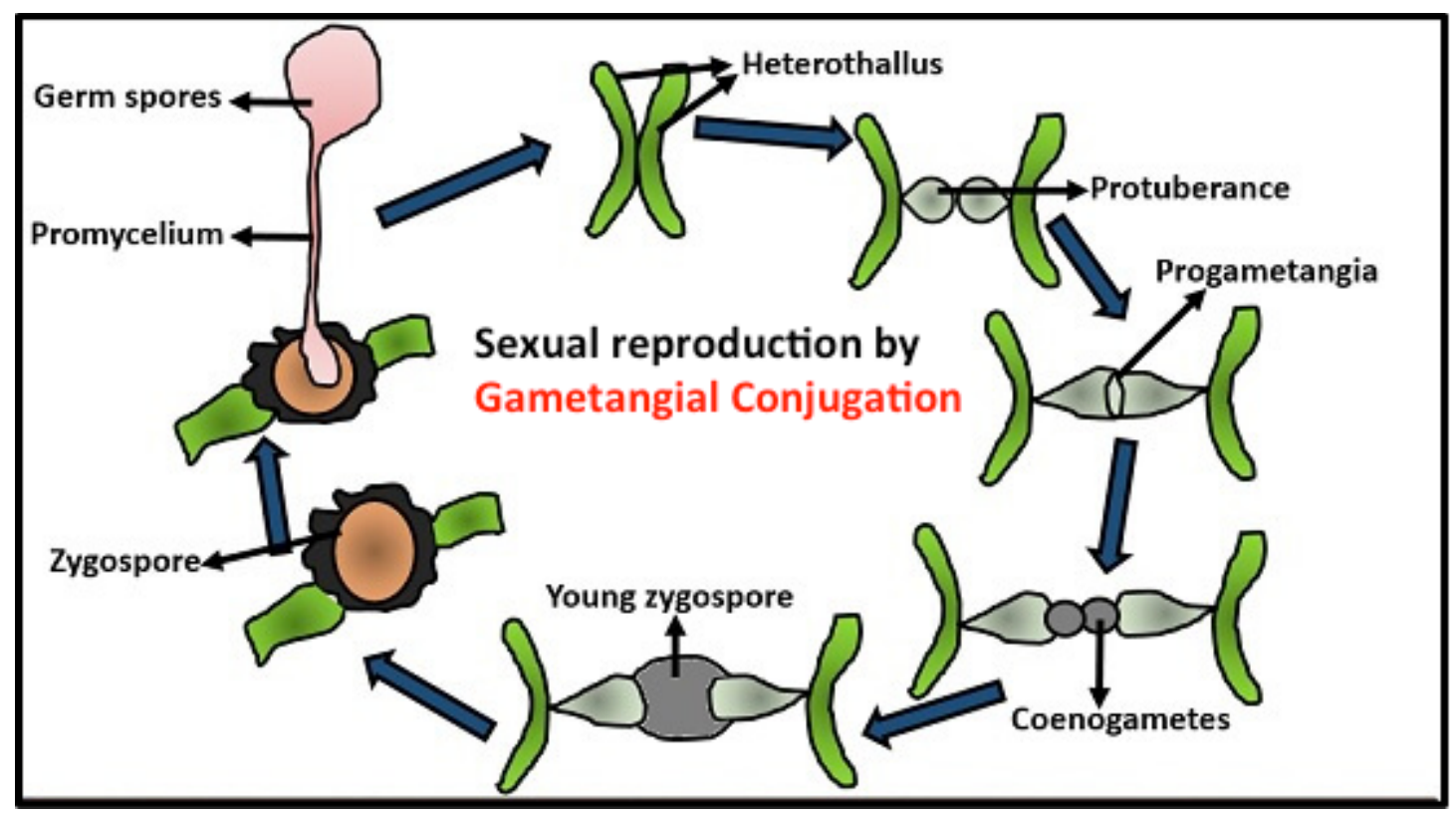

Fig. 6. Sexual reproduction in Mucor species by gametangial conjugation (Supriya 2021b).

Zygophores are produced by two parallel vertical hyphae, which bear outgrowths on the corresponding wall known as progametangium. Sizeincrement and opposite push force happen in progametangia, which results into the formation of suspensor (basal, sterile and more vacuolated part), and 
gametangium (terminal, fertile and more nucleated part). Multi-nucleate and undi-fferentiated protoplast of the gametangium represents a gamete called coenogamete or aplanogamete.

The fusion of coenogamete involves first plasmogamy and then karyogamy and thereby yields diploid zygote. Later, thick wall surrounds the zygote, which is converted into warty, dark black and 2-layered structure known zygospore, containing synkaryon. Within the zygospore, synkaryon under goes meiotic division, thereby form 4 haploid nuclei. Only one surviving nucleus germinates into promycellium, a hyphae-like structure. In turn, the promycellium ends in a columella-lacking sporangium, which produces spores. Finally, new somatic hyphae are produced when each spore germinates (Majumdar and Banerjee 2000).

\subsection{Cunninghamella bertholletiae}

Among Zygomycetes and the Cunninghamellaceae family, Cunninghamella bertholletiae is known to be the only clinically relevant species. However, $C$. bertholletiae is found to be associated with the highest overall mortality (98\%) in immunocompromised hosts. This is because C. bertholletiae causes more aggressive, refractory, and fatal infections despite antifungal therapy (Simitsopoulou et al. 2010; Gomes et al. 2011).

\subsection{Apophysomyces elegans}

The genus Apophysomyces belongs to Division - Zygomycota, Class Mucoromycotina, Order - Mucorales and Family -Saksenaeaceae. Commonly found in soil and decaying vegetation mostly in tropical to subtropical regions, Apophysomyces is a genus of filamentous fungi and historically was monospecific, containing only the type species Apophysomyces elegans (Collier et al. 1998). 
A. elegans species complex comprises A. elegans, A variabilis, A. ossiformis, A. trapeziformis, and A. mexicanus. First isolated from the soil in India in 1979, A. elegans is thermotolerant species and a relatively newer causative agent of mucormycoses in order Mucorales (Misra et al. 1979; Chakrabarti et al. 2003). A. elegans causes mostly cutaneous infections after injury to the skin (Meis and Chakrabarti 2009). Notably, ranking second after Rhizopus arrhizus, Apophysomyces species complex is the rare mucoraceous fungus causing mucormycosis in India, and accounts for nearly $60 \%$ of the reported cases worldwide (Prakash et al. 2016).

\subsection{Lichtheimia (Absidia)}

The zygomycete genus Lichtheimia (syn. Absidia pro parte, Mycocladus), currently contains five mostly saprophytic plant decaying and soil-borne species including Lichtheimia corymbifera (syn. Absidia corymbifera, Mycocladus corymbifer), $L$. ramosa (syn. Absidia ramosa, Mycocladus ramosus) and L. ornata. L. corymbifera is the principal pathogen causing human and animal infections (AlastrueyIzquierdo et al. 2010; Mycology Online 2021a).

\subsection{Saksenaea}

The genus Saksenaea belongs to Division - Zygomycota, Class - Zygomycetes, Order -Mucorales and Family -Saksenaeaceae. Based on physiological, morphological, and molecular characteristics 4 species of the genus Saksenaea (namely S. vasiformis, S. erythrospora, S. oblongispora, and S. loutrophoriformis) have been identified. The filamentous fungus and the primary causative agent of cutaneous mucormycosis Saksenaea vasiformis was first isolated from soil in India by Saksena in 1953. Later, S. vasiformis was isolated from soil samples, driftwood, and grains, from different parts of the world including India, United States, Central America, and Israel (Sigera et al. 2018). S. oblongispora is characterized by oblong sporangiospores and unable to grow at $42^{\circ} \mathrm{C}$, and $S$. 
erythrospora is characterized by large sporangiophores and sporangia and by ellipsoid sporangiospores, biconcave in the lateral view (Alvarez et al. 2010).

\subsection{Rhizomucor pusillus}

Rhizomucor belongs to order Mucorales and family Lichtheimiaceae. The genus Rhizomucor contains six species including $R$. endophyticus, $R$. miehei, $R$. pakistanicus, R. pusillus, R. tauricus, and R. variabilis. A good producer of amylase and a hot environment-loving fungus, Rhizomucor pusillus is cosmopolitan and a thermophilic fungus that exhibits its optimum growth in the temperature range between 50 and $70^{\circ} \mathrm{C}$. The presence of stolons, rhizoids, and branched sporangiophores are peculiar features of R. pusillus cells. In fact, the presence of stolons and poorly developed rhizoids at the base of the sporangiophores help the genus Rhizomucor in distinguishing it from the genus Mucor. Regarding the role of R. pusillis in human diseases, it can lead to mucormycosis/ zygomycosis in humans with a weakened immune system (Mycology Online 2021b; Wikipedia 2021).

\section{Infection-misnomers based on fungi colour}

Mucormycosis represents a group of fungal infections caused by members of the subphylum Mucoromycotina and the order Mucorales. Mucormycosis is also called as 'black fungus' infection. Actually, the black colour does not represent the colour of the fungus; instead, the black colour is the colour of the dead tissues resulting from the fungal infection that invades the blood vessels and destroys them causing tissue death.

Another fungal (Candida)-linked infection (candidiasis) has also caused a stir at the current moment. Candidiasis, the most common fungal infection is being currently called 'white fungus' which is just a myth and misconception. The White fungus infection begins from the tongue or private parts, due to which it turns the tongue white. Since candidiasis affects vital organs including the lungs, 
brain, kidney and private parts along with the mouth, stomach and skin, it is has been argued as more dangerous than infection caused by the black fungus (mucormycosis).

The cases of another fungal infection supposedly caused by the yellow fungus has also been reported from some regions in India. However, the experts has warned the labeling the same fungus with names of different colours, that can create confusion among the people. In fact, the yellow fungus (or Mucor septicusI), is a fungal infection that generally does not occur in humans; however, this infection occurs in reptiles like lizards. Notably, the natural hosts of the yellow fungus are a wide range of lizard species, crocodiles and humans (DH Web Desk 2021).

There exists a great controversy regading the scientific use of the term 'yellow fungus diease'. The term 'yellow fungus disease' for Onygenalean dermatomycoses, (formerly snake fungal disease) in reptiles has been considered obsolete, inaccurate and misleading. In culture, the colour of the actual fungus is white, not yellow. It was suggested to ascribe a specific fungus to an infection (Paré et al. 2021).

\section{Conclusion and perspective}

Prevalent in indoor and occupational environments, most members of the Order Mucorales and the Mucoromycotina subphylum are saprophyte fungal species well-known for causing serious health problems and serious infections including mucormycosis. Though mucormycoses are uncommon but often dramatic, and thus, require immediate action on the basis of an accurate diagnosis. Studies have confirmed that the majority of the cases of mucormycoses (70 to 80\%) involved the Mucoralean members including Rhizopus, Mucor, and Lichtheimia (formerly Absidia) species. On the other, less than 1 to 5\% of reported cases of mucormycosis involved Cunninghamella, Apophysomyces, Saksenaea, Rhizomucor, Cokeromyces, Actinomucor, and Syncephalastrum species. The major outcomes of 
discussion in this paper may help in further widening the botanical views of the readers on the major causative agents of the fatal mucormycoses. However, further exploration of the country-wise data for mucormycosis is warranted that will help in estimation of disease burden in different risk groups, analyse the clinical disease pattern and identify the new etiological agents. Much molecular genetic studies should be conducted to unveil more insights into the potential fatal consequences of mucormycoses in human/ immunocompromised hosts (Prakash and Chakrabarti 2019).

\section{References}

Agrawal R, Yeldandi A, Savas H, Parekh ND, Lombardi PJ, Hart EM (2020) Pulmonary mucormycosis: risk factors, radiologic findings, and pathologic correlation. Radio Graphics 40(3): 656-66

Alastruey-Izquierdo A, Hoffmann K, de Hoog GS, Rodriguez-Tudela JL, Voigt K, Bibashi E, Walther G (2010) Species recognition and clinical relevance of the zygomycetous genus Lichtheimia (syn. Absidia pro parte, Mycocladus). J Clin Microbiol 48(6): 2154

Alvarez E, Garcia-Hermoso D, Sutton DA, Cano JF, Stchigel AM, Hoinard D, Fothergill AW, Rinaldi MG, Dromer F, Guarro J (2010) Molecular phylogeny and proposal of two new species of the emerging pathogenic fungus Saksenaea.J Clin Microbiol 48(12): 4410

Bio Libretexts (2021) Fungi as Plant, Animal, and Human Pathogens, March 6, 2021), https://bio.libretexts.org/@go/page/13642, accessed on May 30, 2021

Blackwell M (2011) The Fungi: 1, 2, 3... 5.1 million species? American J Bot 98(3): 426-438

Botha A, Botes A (2014) Mucor. Encycloped Food Microbiol 2: 834-840

Botha A, du Preez JC (1999) Mucor. Encycloped Food Microbiol 1493-1500

Chakrabarti A, Ghosh A, Prasad GS, David JK, Gupta S, Das A, Sakhuja V, Panda NK, Singh SK, Das S, Chakrabarti T (2003) Apophysomyces elegans: an emerging zygomycete in India. J Clin Microbiol 41(2): 783-788

Chakrabarti A, Singh R (2014) Mucormycosis in India: unique features. Mycoses 57: 85-90

CNN Health (2021) https://edition.cnn.com/interactive/2020/health/coronavirus-maps-andcases/, accessed on 25 May 2021

Collier L, Balows A, Sussman M (1998) Topley \& Wilson's Microbiology and Microbial Infections, $9^{\text {th }}$ ed., vol. 4. Arnold, London, Sydney, Auckland, New York

Dannaoui E, Millon L (2014) Current status of diagnosis of mucormycosis: update on molecular methods. Curr Fungal Infect Rep 8: 353-359

DH Web Desk (2021) MAY 25 2021. Covid-19: All you need to know about 'yellow fungus', https://www.deccanherald.com/national/covid-19-all-you-need-to-know-about-yellowfungus-989747.html; accessed on 27 th May 2021

Gomes MZ, Lewis RE, Kontoyiannis DP (2011) Mucormycosis caused by unusual mucormycetes, non-Rhizopus, -Mucor, and -Lichtheimia species. Clin Microbiol Rev 24(2): 411-445

Hariharan G, Prasannath K (2021) Recent advances in molecular diagnostics of fungal plant pathogens: a mini review. Front Cell Infect Microbiol 10: 600234

Kaushik MP (2014) Fungi. Modern Botany, Prakash Publications, Muzaffar Nagar (U.P.), Reprint 2014, pp. 133-136

Kharayat BS, Singh Y (2018) Mycotoxins in foods: mycotoxicoses, detection, and management. In: Microbial Contamination and Food Degradation, Academic Press, pp. 395-421 
Kontoyiannis DP, Lewis RE (2016) Agents of Mucormycosis and Entomophthoramycosis. In: Mandell, Douglas, and Bennett's Principles and Practice of Infectious Diseases, $8^{\text {th }}$ ed., 2: 2909-2919.e3

Lutzoni F, Miadlikowska J (2009) Lichens. Curr Biol 19: R502-R503

Majumdar NR, Banerjee NN (2000) Fundamentals of Botany. $6^{\text {th }}$ ed., Good Books, Patna, Bihar, India, p. 348

Meis JF, Chakrabarti A (2009) Changing epidemiology of an emerging infection: zygomycosis. Clin Microbiol Infect Suppl 5: 10-14

Misra PC, Srivastava KJ, Lata K (1979) Apophysomyces, a new genus of the Mucorales. Mycotaxon 8: 377-382

Mycology Online (2021a) THE UNIVERSITY OF ADELAIDE, SA 5005 AUSTRALIA, https://mycology.adelaide.edu.au/descriptions/zygomycetes/lichtheimia/\#: :text=The $\% 2$ 0genus $\% 20$ Lichtheimia $\% 20$ currently $\% 20$ contains,often $\% 20$ misidentified $\% 20$ morphologic ally\%20as\%20L, accessed on 6 June 2021

Mycology Online (2021b) THE UNIVERSITY OF ADELAIDE, SA 5005 AUSTRALIA, https://mycology.adelaide.edu.au/descriptions/zygomycetes/rhizomucor/, accessed on 6 June 2021

NDTV (2021) https://www.ndtv.com/india-news/coronavirus-11-717-cases-of-black-fungusso-far-in-india-says-health-ministry-2449502, accessed on 25 May 2021

Paré JA, Wellehan J, Perry SM, Scheelings TF, Keller K, Boyer T (2021) Onygenalean Dermatomycoses (formerly yellow fungus disease, snake fungal disease) in reptiles. $J$ Herpetol Med Surg 30(4): 198-209

Prakash H, Chakrabarti A (2019) Global Epidemiology of Mucormycosis. J Fungi (Basel) 5(1): 26

Prakash H, Ghosh AK, Rudramurthy SM, Paul RA, Gupta S, Negi V, Chakrabarti A (2016) The environmental source of emerging Apophysomyces variabilis infection in India. Sabouraudia 54(6): 567-575

Revankar SG (2021) Aspergillosis. MSD Manual. Merck Sharp \& Dohme Corp., a subsidiary of Merck \& Co., Inc., Kenilworth, NJ, USA; https://www.msdmanuals.com/enin/professional/infectious-diseases/fungi/overview-of-fungal-infections, accessed on 27 May 2021

Sigera LSM, Gamage KKK, Jayawardena MN, Abeydeera WPH, Malkanthi MA, Jayasekera PI, Patabendige CGUA, Fernando AHN (2018) Cutaneous mucormycosis caused by Saksenaea vasiformis in a patient with systemic lupus erythematosus. Clin Case Rep 6(9): 1730-1734

Simitsopoulou M, Georgiadou E, Walsh TJ, Roilides E (2010) Cunninghamella bertholletiae exhibits increased resistance to human neutrophils with or without antifungal agents as compared to Rhizopus spp. Medical Mycol 48(5): 720-724

Skiada A, Pavleas I, Drogari-Apiranthitou M (2020) Epidemiology and diagnosis of mucormycosis: an update.J Fungi (Basel) 6(4): 265 https://doi.org/10.3390/jof6040265

Smith MR., Davis RL (2016) Mycetismus: a review. Gastroenterol Rep 4(2): 107-112 https://doi.org/10.1093/gastro/gov062

Supriya N (2021a) Rhizopus. Biology Reader. https://biologyreader.com/rhizopus.html, accessed on 6 June 2021.

Supriya N (2021b) Mucor. Biology Reader. https://biologyreader.com/mucor.html, accessed on 6 June 2021.

Tan GSE, Seng TT (2018) Overview of fungal infections. Reference Module in Biomedical Sciences doi:10.1016/B978-0-12-801238-3.98320-X

WHO (2018) Mycotoxins. https://www.who.int/news-room/factsheets/detail/mycotoxins\#: :text=Mycotoxins\%20are\%20naturally\%20occurring\%20toxi ns,under\%20warm\%20and\%20humid\%20conditions, accessed on 30 May 28, 2021

Wikipedia (2021) Rhizomucor pusillus, https://en.wikipedia.org/wiki/Rhizomucor_pusillus, accessed on 6 June 2021 